\title{
RANGE OF NICU PRACTICE IN ENGLAND AND WALES REGARDING THRESHOLDS FOR NEONATAL CONJUGATED HYPERBILIRUBINAEMIA AND RELEVANT INVESTIGATIONS
}

\author{
C. Tzivinikos ${ }^{1}$, S. Narayanan ${ }^{2}$ \\ ${ }^{\text {I}}$ Paediatrics, Luton and Dunstable Hospital, Luton, ${ }^{2}$ Paediatric Department, Watford General Hospital, \\ Watford, UK
}

Background: Conjugated jaundice is a common problem in a neonatal intensive care. However, several investigations are performed to exclude underlying liver disease. Opinion differs on their diagnostic value.

Aim: To evaluate practice related to investigation of conjugated jaundice

Method: Questionnaire survey of lead neonatal consultants from all neonatal units in England and Wales.

Results: 102/194 neonatal units (52\%), responded to the survey of which 33 were level 3 units, 50 level 2 and 19 level 1 units. 96 units (94\%) performed conjugated jaundice screen and 6 units $(6 \%)$ did not. 77 units (75\%) had a written policy. $49 \%$ of responders defined conjugated jaundice as conjugated bilirubin $>20 \%$ of total bilirubin and $46 \%$ as $>15 \%$ of total bilirubin and $5 \%$ of units did not have a clear definition. Conjugated bilirubin levels that prompted investigations varied between units with $28(30 \%)$ using conjugated bilirubin $>20 \%$ of total, $33(36 \%)$ a conjugated bilirubin $>15 \%$ of total and $20(21 \%)$ with no definite threshold. Majority $(>76 \%)$ of units performed liver and thyroid function tests, Galactosaemia screen, $\alpha-1$ antitrypsin and liver ultrasound. $65 \%$ of units performed urine culture and hepatitis serology, $32 \%$ performed urine organic acids, NH3 and lactate. 19 units performed CF genetics and 23 HIDA scan. $71 \%$ of responders thought 'diagnostic yield' was 'poor' and $44 \%$ based this on their personal view, $29 \%$ on local data and $27 \%$ on anecdotal evidence.

Conclusion: Our study identified a wide variation in definition and investigation of neonatal conjugated jaundice. National consensus guidelines are required to standardize practice. 